Journal of

Synchrotron

Radiation

ISSN 1600-5775

\title{
Model-independent structure factors from powder X-ray diffraction: a novel approach
}

\author{
Tine Straasø, Ann-Christin Dippel, Jacob Becker and Jens Als-Nielsen
}

J. Synchrotron Rad. (2014). 21, 119-126

Copyright (C) International Union of Crystallography

Author(s) of this paper may load this reprint on their own web site or institutional repository provided that this cover page is retained. Republication of this article or its storage in electronic databases other than as specified above is not permitted without prior permission in writing from the IUCr.

For further information see http://journals.iucr.org/services/authorrights.html

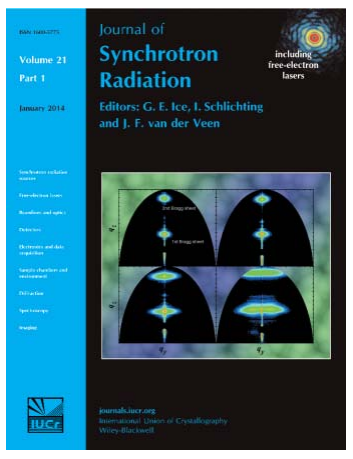

Synchrotron radiation research is rapidly expanding with many new sources of radiation being created globally. Synchrotron radiation plays a leading role in pure science and in emerging technologies. The Journal of Synchrotron Radiation provides comprehensive coverage of the entire field of synchrotron radiation research including instrumentation, theory, computing and scientific applications in areas such as biology, nanoscience and materials science. Rapid publication ensures an up-to-date information resource for scientists and engineers in the field.

Crystallography Journals Online is available from journals.iucr.org 
Journal of

Synchrotron

Radiation

ISSN 1600-5775

Received 19 July 2013

Accepted 14 October 2013

\section{Model-independent structure factors from powder $\mathrm{X}$-ray diffraction: a novel approach}

\author{
Tine Straasø, ${ }^{\mathrm{a}}$ Ann-Christin Dippel, ${ }^{\mathrm{b}}$ Jacob Becker $^{\mathrm{c}}$ and Jens Als-Nielsen ${ }^{\mathrm{a} *}$

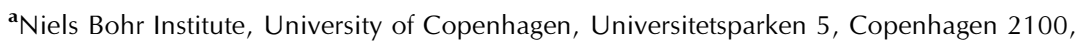 \\ Denmark, 'bPETRA III, Deutches Elektronen-Synchrotron DESY, Notkestrasse 85, Hamburg 22607, \\ Germany, and ${ }^{\mathrm{c}}$ Department of Chemistry and iNANO, Center for Materials Crystallography, \\ Langelandsgade 140, Aarhus 8000, Denmark. *E-mail: als@fys.ku.dk
}

Under the experimental condition that all Bragg peaks in a powder X-ray diffraction (PXRD) pattern have the same shape, one can readily obtain the Bragg intensities without fitting any parameters. This condition is fulfilled at the P02.1 beamline at PETRA III using the seventh harmonic from a $23 \mathrm{~mm}$-period undulator $(60 \mathrm{keV})$ at a distance of $65 \mathrm{~m}$. For grain sizes of the order of $1 \mu \mathrm{m}$, the Bragg peak shape in the PXRD is entirely determined by the diameter of the capillary containing the powder sample and the pixel size of the image plate detector, and consequently it is independent of the scattering angle. As an example, a diamond powder has been chosen and structure factors derived which are in accordance with those calculated from density functional theory methods of the WIEN2k package to within an accuracy that allows a detailed electron density analysis.

(C) 2014 International Union of Crystallography

\section{Introduction}

$\mathrm{X}$-ray diffraction is of basic interest for physical chemistry because the small deviations of the electron density around each atom from spherical symmetry, reflecting the chemical bond between atoms, can in principle be determined by the intensities of X-ray Bragg reflections from a single crystal (see, for example, Coppens, 1997).

However, single-crystal crystallography suffers in this aspect from a number of corrections that must be assessed very accurately before a reliable electron density map can be obtained. These corrections include absorption in the sample, extinction attenuation from the low-angle strong reflections, as well as small scale factor fluctuations between the numerous frames required for a complete diffraction pattern.

None of these drawbacks are present in powder X-ray diffraction (PXRD), which can be thought of as single-crystal diffraction from a huge number of randomly oriented crystal grains that are so tiny that absorption and extinction effects within a grain can be neglected. In addition, the entire diffraction pattern is obtained in one single exposure or frame. Indeed, Nishibori et al. (2007) and Svendsen et al. (2010) recently demonstrated that synchrotron powder diffraction can provide accurate structure factor values and could be the method of choice when dealing with high-symmetry inorganic crystals.

However, when it comes to measuring the deviation from spherical symmetry of the atomic electron densities there is an unfortunate degeneracy in the PXRD pattern, which can most easily be explained by an example. Suppose the crystal has cubic symmetry. Then, for example, the $(3,3,3)$ and the $(5,1,1)$ reflections will both occur at the same scattering angle because $\left(h^{2}+k^{2}+l^{2}\right)$ has the same value of 27 for both reflections. From a single-crystal study the intensity of the two reflections might differ by a small amount and thus directly signify a real deviation from spherical symmetry of the atomic electron cloud, but in PXRD one observes inevitably the weighted sum of the degenerate reflections, weighted by the multiplicity of the respective $(h, k, l)$ sets as well as by their different structure factors, so the direct manifestation of the asymmetric cloud density is lost for such degenerate reflections.

With the present instrument and beam quality one can deal rationally with a number of features in PXRD which conventionally is dealt with by empirical consensus (see, for example, McCusker et al., 1999). The first of these features is reliable background subtraction. In standard program packages this is typically performed by fitting, for example, Legendre polynomials up to 12 th order. This approach is not based on any physical reality and only serves to smooth out data for which the origin is not understood or determined otherwise. As will be shown we have accounted for the background quantitatively by an absolute determination of the Compton scattering, presuming the absence of amorphous phase in the powder. Technically this was feasible by using an all-in-vacuum diffractometer as discussed in a previous paper (Straasø et al., 2013). Next there is the proper integration of the Bragg peaks when dealing with peak widths that vary with the scattering angle. Here it is a common procedure to simu- 
late the peak shape by an analytic function with a number of adjustable parameters, for example a pseudo-Voigt profile with five parameters that have little bearing in physical reality, although some authors have synthesized the line profile by convoluting components which could be assessed individually (e.g. Cheary \& Coelho, 1992; Straas $\emptyset$ et al., 2013).

In the present study the beam quality in terms of divergence and wavelength band is so good that the powder peak widths and peak shapes are entirely determined by the combination of the capillary diameter and the pixel size, utilizing the fact that the grains are large enough to not contribute to the peak width. Therefore the peak widths and peak shapes are independent of scattering angle. Under these experimental conditions the integrated intensities, even for overlapping peaks, can be obtained by a set of linear equations, the socalled normal equations (see e.g. Taylor, 1997), without any fitting of parameters at all. In the present case of a diamond powder there are no overlapping peaks and the normal equations simplify to just one equation for the scaling of a given reference peak relative to any Bragg peak in the powder pattern. This method of extracting the integrated intensities is a special and simple case of the method discussed by Pawley (1981) using the standard procedure in statistical analysis with normal equations. In our case the normal equation matrix is diagonal with identical diagonal elements that can be derived algebraically without any fitting parameters at all.

The question then remains whether the PXRD data are of sufficient quality to unambiguously reveal electron density deviations from spherical symmetry without relying on any model fitting. We shall show that this is indeed the case, and the example we have chosen is that of diamond because the

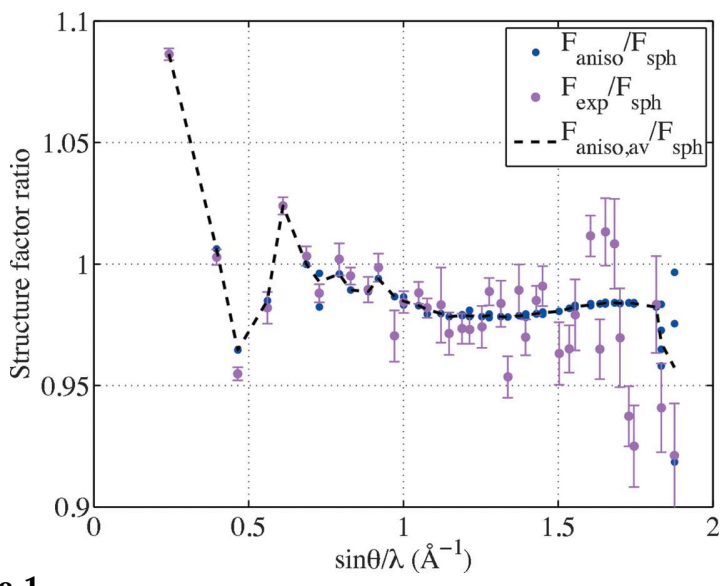

Figure 1

The structure factor ratio between the theoretical aspherical model and the spherical IAM of the electron density in diamond is shown as blue dots. The black dotted curve is a guide to the eye, which in case of degenerate reflections shows the weighted average of the theoretical structure factors. It serves as the theoretical result, which the experimentally derived structure factors can be compared with. Note that the difference between the IAM and the aspherical model is at most a few percent. The structure factor ratio between the derived structure factors from PXRD and the IAM is shown in magenta with error bars as given in Fig. 6. The experimental structure factors were obtained by scaling the reference peak as outlined in \$3.1. Only structure factors originating from Bragg reflections with an observed intensity larger than $5 \%$ of the background level have been included in the analysis. electron density has been calculated with great accuracy for the diamond lattice and therefore the $(h, k, l)$ structure factors are known theoretically with high accuracy. In addition, the thermal vibrations of the electron cloud are rather small and isotropic in diamond, and thermal vibrations can therefore be corrected for reliably to obtain the static structure factors needed for comparison with the theoretical structure factors. As an extra benefit the correction for thermal diffuse scattering is negligible due to the high beam quality, yielding a very small probing volume in reciprocal space.

The deviation from spherical electron density as expressed in the ratio between the calculated structure factors and the spherical independent atom model (IAM) is indeed quite close to unity as shown in Fig. 1 by the dotted black curve. Apart from the $(1,1,1)$ reflection the difference is only a few percent, so to verify this experimentally requires a very precise powder pattern. As can be seen from Fig. 1, in foresight of describing the experimental details, it is indeed possible to obtain this kind of accuracy. The perspective is then that PXRD can determine such tiny deviations, also in cases where theoretical calculations are not available.

\section{Experimental details}

\subsection{Theoretical computation}

Theoretical static structure factors published by Bindzus et al. (2014) have been used to evaluate the experimentally derived structure factors. The theoretical electronic structure was determined using the density functional theory (DFT) methods of the WIEN2k package (Blaha et al., 2008).

\subsection{Data collection}

Crystalline large-grain $(2-4 \mu \mathrm{m})$ diamond powder was purchased from Nilaco and packed in a $0.2 \mathrm{~mm}$ glass capillary. A diamond grain with a diameter $D$ of $2 \mu \mathrm{m}$ may be considered as a perfect crystallite with no secondary extinction. The primary extinction coefficient $E$ is very close to unity. ${ }^{\mathbf{1}}$ In order to facilitate high-resolution powder X-ray diffraction to large reciprocal lattice vectors, data were collected at the $60 \mathrm{keV}$ P02.1 beamline (Dippel et al., 2014) at the PETRA III synchrotron, Germany, using an all-in-vacuum diffractometer, the details of which are described by Straas $\varnothing$ et al. (2013). In brief, all air scattering is eliminated by keeping the sample and the image plate (IP) detector in the same vacuum. In such a set-up the background level will be at the fundamental level consisting of only the scattering from the sample holder and the inevitable Compton scattering from the sample itself. A semi-transparent beam stop of $3.5 \mathrm{~mm}$ tantalum provides a precise location on the IP of the direct beam. A wavelength of $\lambda=0.20687$ (2) $\AA$ was determined by the symmetric positions of Bragg reflections from single crystals of $\mathrm{Si}(a=5.4309 \AA)$ both in Laue and Bragg geometry. The raw two-dimensional

\footnotetext{
${ }^{1}$ From International Tables of Crystallography (1992), Vol. C, §6.4.8, one has $E=1-x / 2 \ldots$, with $x=\left(N_{\mathrm{c}} \lambda D r_{0} F_{h k l}\right)^{2}$, yielding $E=0.999$ for the $(1,1,1)$ reflection, $N_{\mathrm{c}}$ being the inverse of the unit-cell volume and $r_{0}$ the Thomson scattering length.
} 
diffraction image is integrated along the exact DebyeScherrer rings as explained by Straas $\varnothing$ et al. (2013) to yield the final one-dimensional powder diffraction pattern. Referring to Fig. 11 in that paper we have numerically examined the effect of the finite horizontal beam width, i.e. that the location of point ' $\mathrm{O}$ ' in that figure is smeared. Usually this effect is referred to as axial divergence and it has been dealt with in the literature by van Laar \& Yellon (1984) and Cooper \& Glasspool (1976). Our numerical study shows that in the present study the effect is utterly negligible.

\subsection{Beam characteristics and peak shape}

The X-ray source is a long undulator, $L=2 \mathrm{~m}$, with a period of $\lambda_{\mathrm{U}}=23 \mathrm{~mm}$. A narrow wavelength band around $60 \mathrm{keV}$ is extracted from the seventh harmonic in the undulator spectrum by two successive Bragg reflections in the horizontal plane from first a diamond(1,1,1) crystal and second from a $\mathrm{Si}(1,1,1)$ crystal, in asymmetric and symmetric Laue geometry, respectively. The beam divergence from the seventh harmonic is exceedingly small, of order $(\lambda / L)^{1 / 2} \cong 3 \mu \mathrm{rad}$ when neglecting the electron beam divergence. By performing slit scans the X-ray beam dimensions were measured at $s=62.5 \mathrm{~m}$ downstream of the source. FWHM values in millimeters were $w_{\mathrm{h}}=0.57$ in the horizontal direction and $w_{\mathrm{v}}=1.2$ in the vertical direction corresponding to divergences of $\delta_{\mathrm{h}}=w_{\mathrm{h}} / s=9.1 \mu \mathrm{rad}$ and $\delta_{\mathrm{v}}=w_{\mathrm{v}} / s=19.2 \mu \mathrm{rad}$, respectively. The wavelength band is around $2 \times 10^{-4}$ (Dippel et al., 2014). Rocking curves from single crystals of $\mathrm{Si}$ in Laue and Bragg geometry yielded the $(h, h, h)$ widths shown in Fig. 2, with the expected widths given by the red curve. For the powder diffraction experiment the incident beam was apertured down to $0.5 \mathrm{~mm} \times 0.3 \mathrm{~mm}$ (horizontal $\times$ vertical), so the expected rocking-curve widths, and therefore also the powder line widths, are reduced to be on the blue curve. As we shall see in $\$ 2.3 .1$, the contribution to

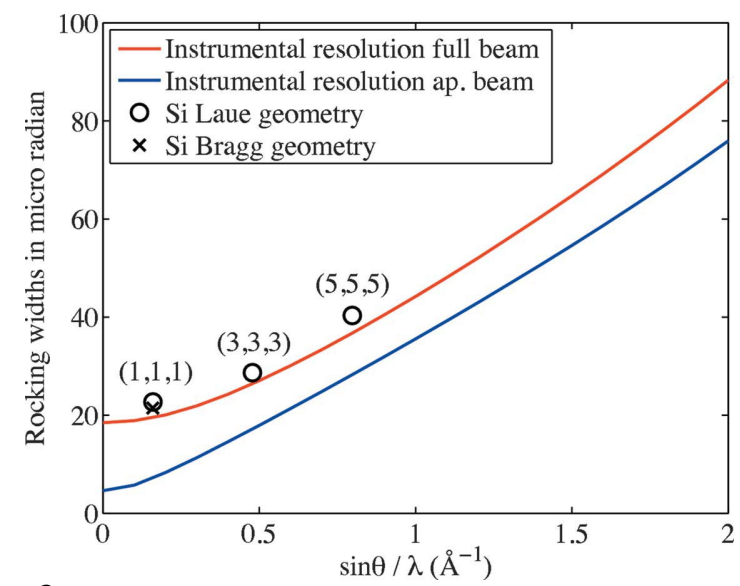

Figure 2

Instrumental resolution, i.e. resolution in reciprocal space from components upstream of the sample position. The red line shows the expected rocking-curve width from a Si single crystal irradiated by the full beam. Experimentally observed rocking widths for one to three $(h, h, h)$ reflections in Bragg geometry (crosses) and Laue geometry (circles), respectively, are in good accordance with the expected values. XRPD data were obtained with an apertured beam leading to the instrumental resolution represented by the blue line. the powder peak width from the capillary diameter of $0.2 \mathrm{~mm}$ at a distance of $R=300 \mathrm{~mm}$ from the IP is $\delta_{\text {cap }}=0.2 \mathrm{~mm} /$ $300 \mathrm{~mm}=670 \mu \mathrm{rad}$, unambiguously proving that all contributions to the powder line width from components upstream of the sample position are negligible.

2.3.1. Powder Bragg peaks. When the beam is Bragg reflected from a strain-free powder sample there will in principle be additional peak broadening due to the finite grain size, $D$, and the finite sample height, $h$. The grain size contribution is given by Scherrer's formula as $\lambda /(D \cos \theta)=$ $20 \mu \mathrm{rad} / \cos \theta$. The contribution to the peak broadening from the finite sample height has no angular dependence and is given in the preceding paragraph to be $670 \mu \mathrm{rad}$. Allowing for a small wobbling during rotation of the capillary, it is likely that the effective capillary diameter was closer to $250 \mu \mathrm{m}$ than $200 \mu \mathrm{m}$, making this contribution even slightly larger. Finally, the pixel size, $p$, of the IP detector gives a constant contribution to the peak width and amounts to $p / R=25 \mu \mathrm{m} / 0.3 \mathrm{~m}=$ $83 \mu \mathrm{rad}$. By comparing the magnitudes of the various contributions it is clear that all contributions other than the two constant contributions from the finite sample height and detector pixel size are negligible. As a consequence the Bragg peaks in the powder diffractogram will all have the same width, independent of the scattering angle $2 \theta$, and, equally important, they will all have the same shape.

\subsection{Data reduction to integrated intensities}

The diffraction pattern is shown in Fig. 3 together with the signal from a similar glass capillary. It is noted that Bragg peaks out to $2 \theta=50^{\circ}$ corresponding to $\sin \theta / \lambda=2 \AA^{-1}$ are observable and that none are overlapping in the entire region.

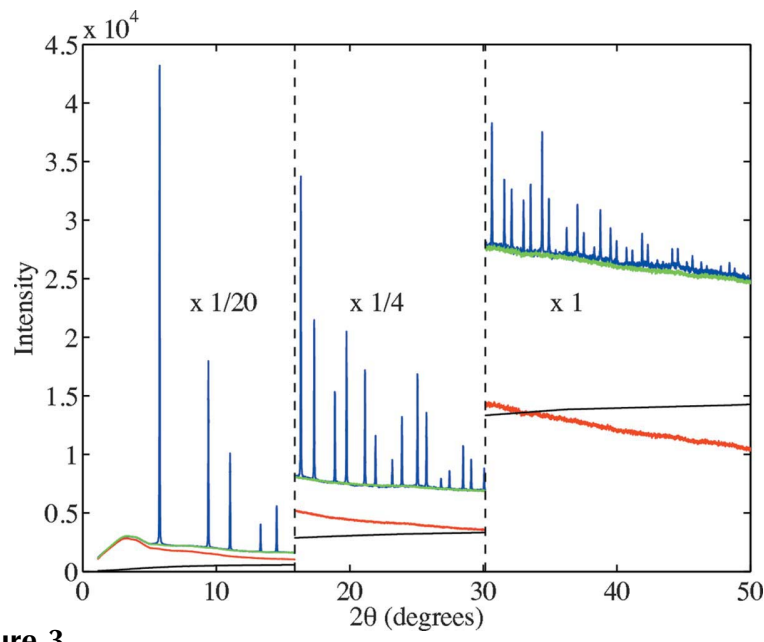

Figure 3

Powder pattern from diamond powder in a $0.2 \mathrm{~mm}$-diameter glass capillary obtained using an all-in-vacuum diffractometer equipped with a curved IP strip as detector. The wavelength was $0.20687 \AA$. The red curve is from an empty glass capillary, and the black curve is the calculated Compton scattering from the diamond powder as explained in the main text. The sum of the red and black curves then amounts to the background below the Bragg peaks as shown in green. Note that a typical peak level of Bragg peaks at large angles is around 2500 counts, so the signal-to-background ratio is around 1:10 at these angles. The exposure time was $60 \mathrm{~min}$. The data set can be found as supporting information, available from the IUCr electronic archives (Reference: GB5017). 
At scattering angles larger than $2 \theta=50^{\circ}$, essentially all scattering from both the diamond powder and the glass capillary is inelastic incoherent Compton scattering [see, for example, International Tables of Crystallography (1992)]. From Fig. 3 one can deduce that Compton scattering from the diamond powder at $2 \theta=50^{\circ}$ is the difference between total scattering of 25000 counts and the glass capillary scattering of 11000 counts, i.e. the Compton scattering from the diamond amounts to about 14000 counts. In International Tables of Crystallography, Vol. C, the ratio of Compton scattering to total scattering is tabulated for all elements $(Z)$ and wavevector transfers, $\sin \theta / \lambda$, so one can generate the Compton scattering from the diamond powder (black curve). The total background shown in green is simply the sum of the signal from the glass and the calculated Compton scattering from the diamond sample. Subtraction of the total background (the green curve) from the diffraction pattern yields the final Bragg scattering data set. However, a fine-tuning may be necessary. The signal from the glass capillary, which is the major contribution to the total background at small scattering angles, was derived from a similar, but not identical, capillary to the one containing the powder. Consequently, the scattering from the two capillaries may differ by a factor close to unity, but not exactly 1 . The appropriate factor of 1.02 was determined from the analysis depicted in Fig. 4.

Moreover, one may fine-tune the background by averaging the remaining intensities between Bragg peaks. This finetuning of the background at high angles was typically around one-tenth of the Bragg peak intensity compared with the full background level which, as apparent from Fig. 3, is ten times higher than the Bragg peak. In other words the fine-tuning of the background was of order $1 \%$ of the total background, as derived by the Compton scattering analysis described above.

Finally we note that the calculated ratio of Compton scattering from glass and from solid diamond is not the ratio of the red and black curves at $2 \theta=50^{\circ}$ in Fig. 3 because of the packing of grains in the capillary, implying that the diamond density inside the capillary is in fact only about $40 \%$ of the density of solid diamond, a typical number for packing of powder.

As emphasized at the end of $\$ 2.3 .1$ we expect the shape of all Bragg peaks to be the same. The proof of this statement, which greatly simplifies the assessment of integrated intensities, is illustrated in Fig. 5, which shows panels of nine different Bragg peaks spread over the entire range of scattering angles. Each $(h, k, l)$ Bragg peak comprising the data points $y_{j}, j=1 \ldots m$, is superimposed on the reference peak,

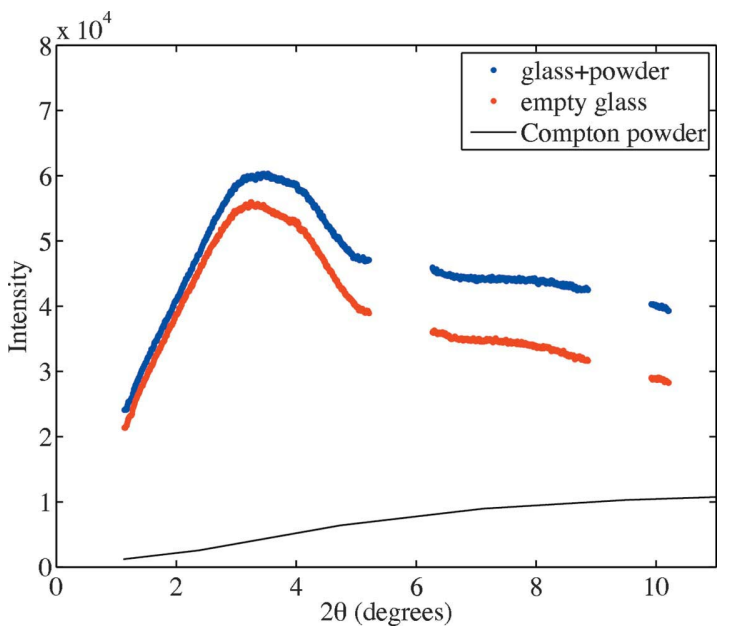

Figure 4

The empty glass capillary is not strictly identical to the capillary containing the diamond powder. Subtracting the Compton scattering from diamond (black curve) from the glass + powder data, where the Bragg peaks at around $5.8^{\circ}$ and $9.3^{\circ}$ have been masked, yields a curve which in shape is identical to the red empty glass curve but due to the different glass differs by a factor of 1.020 .

the latter being displaced to the $(h, k, l)$ scattering angle and denoted $r_{i}, i=1 \ldots n$, and scaled with a certain factor $a^{h k l}$. The intensities of the $(h, k, l)$ Bragg peak are shown with their Poisson error bars $\pm \sigma_{j}$ which at the larger scattering angles mostly originate from the background level. The reference peak could be any peak in the powder diffractogram, but, as
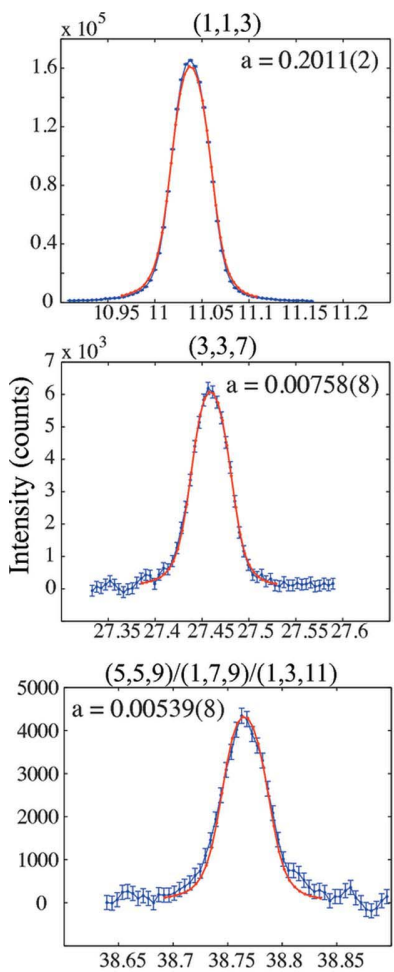

Figure 5
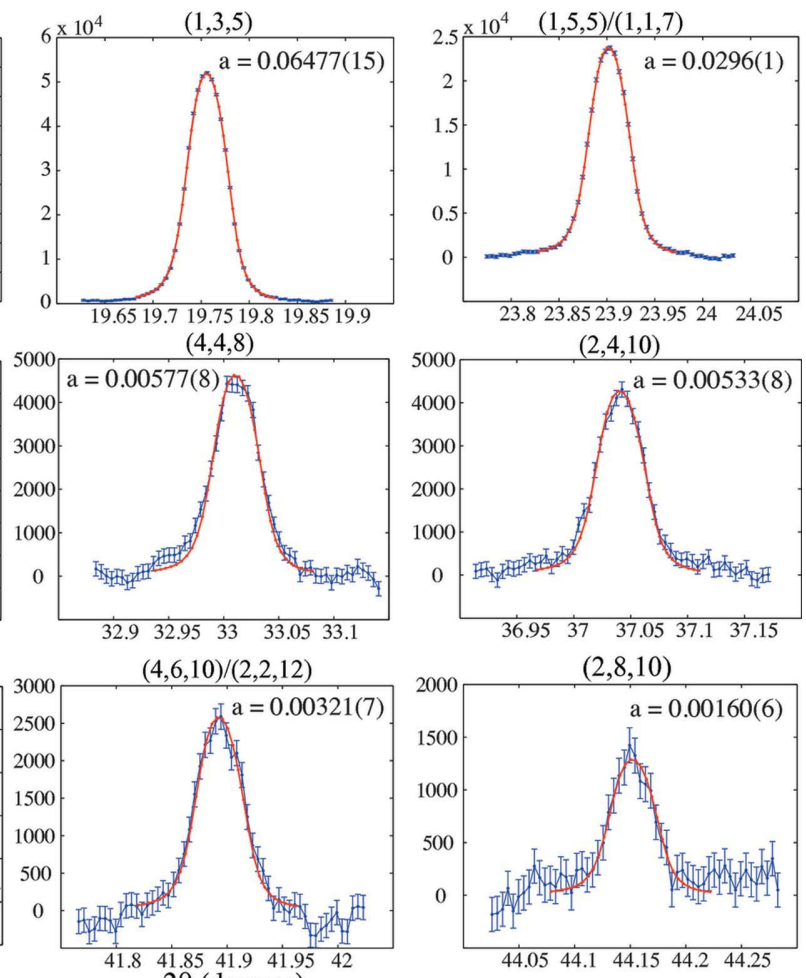

Nine Bragg peaks superimposed on the scaled reference peak shown by a red line prove that all Bragg peaks in the powder spectrum shown in Fig. 3 have the same shape and only differ from each other by a scale factor $a$, which is also given in each panel. Clearly the integrated intensities relative to the reference peak are also given by this scale factor. 
will be further elaborated in the Discussion, the reference peak was chosen as the average profile of the 15 most intense Bragg peaks. The overlap of the $(h, k, l)$ Bragg peak and the scaled reference peak can be quantified by the weighted squared sum of differences, $\chi^{2}$, with the best scale factor $a^{h k l}$. The latter is found by the simple algorithm

$$
a^{h k l}=\sum_{i=1}^{n} w_{i}^{h k l} y_{i}^{h k l} r_{i} / \sum_{i=1}^{n} w_{i}^{h k l} r_{i} r_{i}
$$

Here $y_{i}^{h k l}$ and $r_{i}$ are the intensities of the $(h, k, l)$ reflection and the reference peak, respectively, and the weight of the $i$ th data point is $w_{i}=1 / \sigma_{i}^{2}$. Equation (1) is derived by requiring minimal value of $\chi^{2}=\sum w_{i}^{h k l}\left(y_{i}^{h k l}-a^{h k l} r_{i}\right)^{2}$, so its derivative with respect to $a^{h k l}$ must be zero. The scaling algorithm presented in (1) is a special case of normal equations in statistical analysis, which deals with the more general case of overlapping reflection peaks (see Appendix $B$ ).

The accuracy of the scale factor $a^{h k l}$ due to counting statistics is discussed in Appendix A. However, as pointed out by Ida \& Izumi (2011), the finite number of crystallites will in principle also contribute to the uncertainty of the Bragg intensities, and this effect is particularly pronounced for large crystallite sizes and/or small probing volumes in reciprocal space. Although the illuminated length of the capillary was $0.5 \mathrm{~mm}$ implying that about 360.000 grains were illuminated and the Debye-Scherrer arcs on the IP did not show any individual grain structure, particle statistics might still be an issue. In extending the pioneering work of Alexander et al. (1948) and De Wolff (1959), Ida \& Izumi suggest for a spinning specimen the following relationship between the variance due to particle statistics, $\sigma_{\mathrm{p}}^{2}$, and the effective multiplicity, $m_{\mathrm{eff}}$, given explicitly for the $j$ th data point as

$$
\left(\sigma_{\mathrm{p}}\right)_{j}^{2} \propto\left[y\left(2 \theta_{j}\right)-b_{j}\right]^{2} \sin ^{2} \theta_{j} /\left(m_{\mathrm{eff}}\right)_{j},
$$

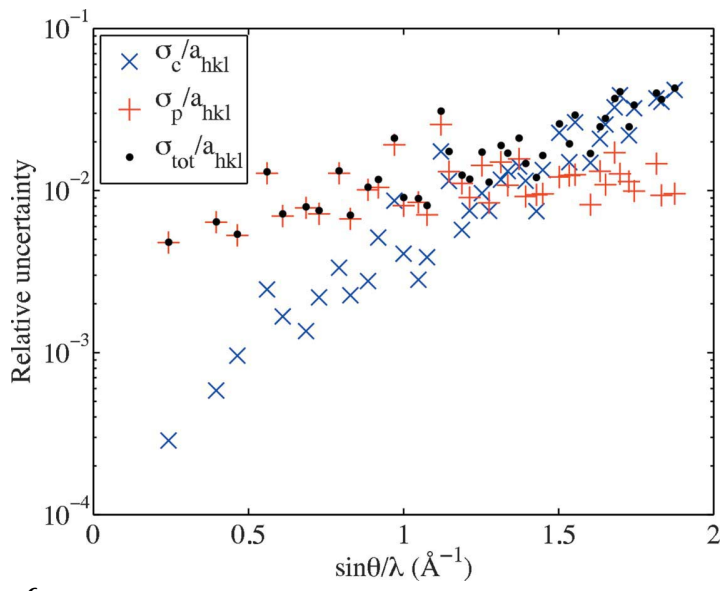

Figure 6

The relative total standard deviation, $\sigma_{\mathrm{t}} / a$, as a function of $\sin \theta / \lambda$ is shown as black dots along with its two components $\sigma_{\mathrm{c}} / a$ and $\sigma_{\mathrm{p}} / a$, where the subscripts $\mathrm{c}$ and $\mathrm{p}$ refer to counting statistics and particle statistics, respectively. At small wavevector transfers the uncertainty of the scale factor $a$ is dominated by particle statistics, whereas at high wavevector transfers the major contributor to the total uncertainty is the counting statistics. where $y\left(2 \theta_{j}\right)$ is the true model intensity and $b_{j}$ is the background intensity. Given that there is no peak overlap in the entire diffraction range, $m_{\mathrm{eff}}$ is simply the (degenerate) multiplicity of a given $(h, k, l)$ reflection. Ida \& Izumi treat the proportionality constant as a refinable parameter during leastsquares refinement. For the present study a proportionality constant of 0.25 is found to return a set of structure factors with the statistically expected Gaussian variance, i.e. about two-thirds of all structure factors below $\sin \theta / \lambda=1.5 \AA^{-1}$ overlap with the theoretical result. At wavevector transfers larger than $\sin \theta / \lambda=1.5 \AA^{-1}$ the total variance is dominated by counting statistics and these data are therefore not included in determining the proportionality constant. Fig. 6 shows the total relative standard deviation alongside the individual contributions from counting and particle statistics, respectively. The total standard deviation was evaluated for all $a^{h k l}$ and used for calculating the error bars in Figs. 1 and 7.

\section{Results}

\subsection{Intensity data reduced to static structure factors}

The intensity versus $2 \theta$ of Bragg peaks, such as the examples shown in Fig. 5, is related to the static structure factor $F_{h k l}$ by

$$
I_{h k l}(2 \theta) \propto\left|F_{h k l}\right|^{2} \exp \left[-2 B(\sin \theta / \lambda)^{2}\right] m_{h k l} \cos \theta \frac{1}{\sin 2 \theta} \frac{1}{\sin 2 \theta} .
$$

In equation (3) $m_{h k l}$ is the multiplicity of the $(h, k, l)$ reflection, $\cos \theta$ is proportional to the circumference of the DebyeScherrer ring, $1 / \sin 2 \theta$ is proportional to the fraction seen by the detector, and the last factor $1 / \sin 2 \theta$ is proportional to the integrated intensity as derived from the Lorentz factor. For degenerate sets of Miller indices $(h, k, l)$ the experimentally observed intensity corresponds to the sum of the intensities from (3) having a common value of $2 \theta$. With no model interpretation, one can only derive the average of structure factors from degenerate reflections.

Thermal vibrations affect the scattered X-ray intensity in two ways, namely by inelastic one-phonon scattering and by diminishing the elastic Bragg scattering by the Debye-Waller factor, $\exp \left[-2 B(\sin \theta / \lambda)^{2}\right]$.

The one-phonon scattering cross section is overlapping the narrow region of Bragg scattering in reciprocal space with a cusp-like intensity profile due to the acoustic phonons. This effect must be corrected for, the so-called thermal diffuse scattering (TDS) correction, and it has been evaluated by Lucas (1969). In the present case the overlap region is very small due to the extremely fine angular resolution of the incident radiation and the narrow wavelength band. Furthermore, in a hard material such as diamond the acoustic phonon energies are increasing rapidly with wavevector and estimates using the Lucas formalism show that the TDS correction in our case is completely negligible. ${ }^{2}$

\footnotetext{
${ }^{2}$ Evaluated using the elastic constants measured by McSkimin \& Andreatch (1972) and $\Delta \theta$ equal to less than $100 \mu \mathrm{rad}$ (contributions from incident collimation, finite crystallite size and incident wavelength band).
} 
Table 1

Reported $B$ values in diamond at room temperature as deduced by various methods.

\begin{tabular}{lll}
\hline Reference & Method & $B\left(\AA^{2}\right)$ \\
\hline Stewart (1973) & Lattice dynamics & $0.151(1) \dagger$ \\
Spackman (1991) & Pendellösung & $0.140(10)$ \\
Fischer et al. $(2011)$ & Powder diffraction & $0.139(4)$ \\
Bindzus et al. $(2014)$ & Powder diffraction & $0.143(1)$ \\
Yamamoto et al. $(1996)$ & Single crystal & $0.164(1)$ \\
This work & Powder diffraction & $0.151(2)$ \\
\hline
\end{tabular}

$\dagger$ Stewart (1973) reports a value of $B=0.149-0.150$, but reproduction of the calculation yields $B=0.151(1) \AA^{2}$.

Parameter $B$ in the Debye-Waller factor is derived from a Wilson plot as shown in Fig. 7, where the logarithm of the ratio of the observed and calculated squared structure factors are plotted versus $(\sin \theta / \lambda)^{2}$. The slope yields a value of $2 B=2 \times$ 0.151 (2) $\AA^{2}$ which can be compared with other reported values for the thermal vibrations in diamond listed in Table 1. An absolute scaling of the experimentally derived structure factors is also provided by the Wilson plot by fixing the fitted line at point $(0,0)$.

With the determination of the Debye-Waller factor the static structure factors can now be calculated and the result is shown in magenta in Fig. 1 expressed as the ratio between the experimentally observed static structure factors, $F_{\text {exp }}$, and those of the spherical IAM, $F_{\mathrm{sph}}$. The relative uncertainty of the observed structure factors as depicted by error bars is half of the relative error on the corresponding scale factors $a^{h k l}$. Out to $\sin \theta / \lambda \simeq 1.5 \AA^{-1}$ there is a clear deviation from the spherical model and the observed structure factors follow the same trend as the theoretical values $\left(F_{\text {aniso }}\right)$. At larger wavevector transfers any trend in the observed structure factors is masked by rather high uncertainties caused by Compton scattering causing signal-to-noise ratios as low as 1:20. Bragg reflections with a signal-to-noise ratio of less than 1:20 have been excluded in the analysis.

In addition to Fig. 1, our results for dynamic structure factors are also listed explicitly in Table 2 . In case of degen-

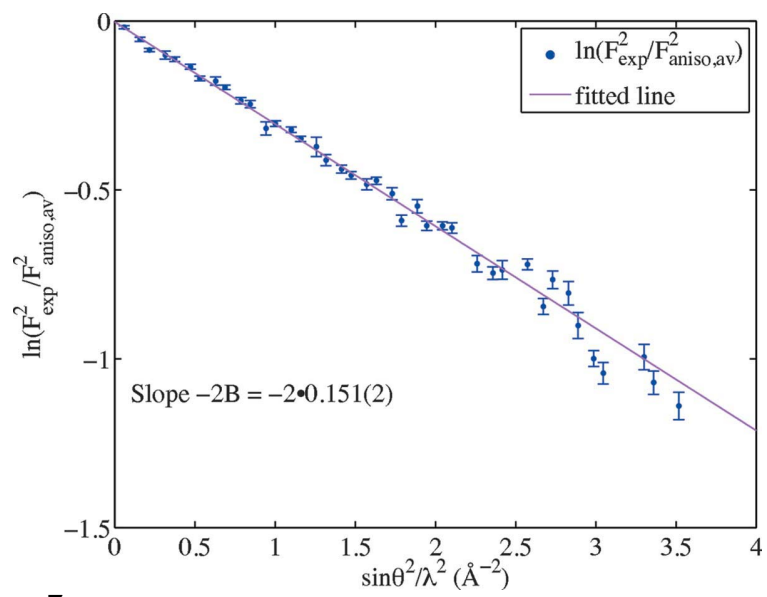

Figure 7

Wilson plot determining the thermal vibrations in diamond. The slope of $-2 B=-0.3025(40) \AA^{2}$ corresponds to an isotropic squared average thermal amplitude of $\left\langle u^{2}\right\rangle=B / 8 \pi^{2}=0.00192$ (3) $\AA^{2}$.
Table 2

Section of observed dynamic structure factors.

Observed dynamic structure factors, i.e. including thermal vibrations. The entire structure factor list can be found as supporting information. Structure factors from degenerate reflections are based on a decomposition of the Bragg intensity according to the ratios of the theoretical contributions as computed by the DFT calculations. Only structure factors derived from Bragg reflections with a signal-to-noise ratio larger than $1: 20$ have been included in the analysis.

\begin{tabular}{|c|c|c|}
\hline$h k l$ & $F_{\text {obs }}$ & Degeneracy \\
\hline 111 & $18.442(44)$ & 1 \\
\hline 022 & $15.364(49)$ & 1 \\
\hline 113 & $9.193(25)$ & 1 \\
\hline 004 & $11.902(77)$ & 1 \\
\hline 133 & $8.344(30)$ & 1 \\
\hline 224 & $10.756(43)$ & 1 \\
\hline 333 & $7.122(27)$ & 2 \\
\hline 115 & $7.222(27)$ & 2 \\
\hline 044 & 9.683 (64) & 1 \\
\hline$\vdots$ & $\vdots$ & $\vdots$ \\
\hline
\end{tabular}

erate reflections the Bragg intensity has been decomposed according to the theoretical structure factors and to the respective multiplicities.

\section{Discussion}

In an ideal data set all peaks could function as the reference peak and different choices would simply change all $a^{h k l}$ values by a constant factor. However, in a real data set the peak shape at large $\sin \theta / \lambda$ is uncertain due to counting statistics, and at small $\sin \theta / \lambda$ there is some uncertainty due to grain statistics (see Fig. 6). The optimal choice of the reference peak shape is an average of the peaks at low $\sin \theta / \lambda$ because the averaging process may even out the uncertainties due to grain statistics and other small unspecified systematic errors. By setting a criterion of a minimum peak intensity of 15000 counts, the error due to counting statistics is less than $1 \%$ and approximately a factor of ten smaller for the integrated intensity. Consequently, as the reference peak we have taken the average of all reflection peaks with a minimum peak intensity of 15000 counts (here 15 ).

In addition there is the choice of how much of the reference beam profile that should be scaled to an $(h, k, l)$ peak. In principle it could be the central point only $(n=1)$, but then one loses the statistical accuracy of including more points. On the other hand, by extending the region too far out in the tails, where the statistical accuracy of the $(h, k, l)$ peak at any rate is low and other systematic effects are relatively more pronounced, one collects in principle relatively more TDS and one may also erroneously include artefacts of irregularities in the background. As shown in Fig. 5 we have chosen a region which is a compromise of the two limiting cases including a portion of the tails $(n=31)$. Changing the number of data points in the reference peak to either $n=41$ or $n=21$ has no effect on the resulting structure factors or estimated thermal parameter within the estimated uncertainties.

The idea to use a numerical profile rather than an anatic mathematical expression was also noted by Hepp \& Baerlocher (1988). 


\section{Summary and outlook}

In papers on crystallography the word 'refinement' occurs ubiquitously. We suggest to distinguish between refinement of the instrument parameters and refinement of the model parameters. On the instrument parameter refinement side, we discussed above the fine-tuning of the background on top of the overall and dominating calculated Compton scattering, as well as the fine-tuning of the scaling between different glass capillaries. Once these instrument corrections are made, the analysis progresses with no fitting of any parameter by using the method of normal equations, outlined in Appendix $B$, to yield a set of weighted structure factors. In the particular case discussed here, i.e. a diamond pattern, there are no overlapping Bragg reflections, so the coefficient matrix $M_{i j}$ in the normal equations is diagonal with identical diagonal elements.

In case of degenerate reflections the obtained structure factor will represent the average of the degenerate structure factors. If one wishes to decompose a degenerate reflection into its various structure factors one may do so according to a model providing their ratios. The obtained structure factors may then serve as the input data to refinement of the model.

We emphasize that the condition for this simple and transparent analysis is that Bragg peak shapes are independent of scattering angle as is the case when the width is completely dominated by the diameter of the capillary.

As far as future improvements are concerned, it is clear from Fig. 1 that it would be a great improvement if the uncertainties at high values of $\sin \theta / \lambda$ could be reduced. As seen in Fig. 3 the background at large scattering angles is due to Compton scattering, and the background X-ray photons therefore have a lower energy than the elastically scattered photons in the Bragg peaks. The background can therefore be dramatically reduced with an energy-dispersive detector instead of an IP detector. Here the incident photon energy of $60 \mathrm{keV}$ is a great benefit, because the relative energy shift in terms of the Compton length $\lambda_{\mathrm{C}}=3.86 \times 10^{-3} \AA$ and the wavevector $k$, namely $\Delta E / E=\lambda_{\mathrm{C}} k(1-\cos 2 \theta)$, is proportional to $E$ and as large as $3.1 \%$ at $2 \theta=30^{\circ}$. We plan to complement the IP detector with an energy-dispersive detector in our set-up in the near future.

\section{Conclusion}

We have shown that the powder pattern obtained on a highly collimated $60 \mathrm{keV}$ beam derived from the seventh harmonic of an undulator source can be analyzed to determine structure factors to an accuracy of $1 \%$ up to $\sin \theta / \lambda=1.5 \AA^{-1}$. The novel analysis is free of any fitted parameters, either for background, for peak shape, for wavelength or for zero scattering angle. The $1 \%$ accuracy required for a model-free derivation of the difference electron density map may be extended to higher values of $\sin \theta / \lambda$ than $1.5 \AA^{-1}$ by supplementing the IP detector with an energy-dispersive detector to eliminate the Compton scattering at these high wavevector transfers.

\section{APPENDIX $A$ \\ Derivation of the statistical weight on different $(h, k, l)$ intensities}

The issue in this Appendix is how to assess the accuracy of the derived scale factors $a$ using equation (1) in $\$ 2.4$. The reference Bragg peak shape is shown in green in Fig. 8 together with the scaled 15 most intense reflections which it is an average of. It represents 31 data points of the total scattering with a background of 25000 counts subtracted. Fig. 9 shows five Poisson-randomized generated peaks with a chosen scale factor of 0.002 , i.e. a peak intensity of about 1500 counts. For simplicity, only one of the data series (in blue) is drawn with error bars including the Poisson statistics of the background level of 25000 counts. In comparison, the error bars on the reference peak are negligible. To gain statistical accuracy, 100 model peaks are generated and the best-fit value of the scale factor $a(i), i=1 \ldots 100$, is found. From that data set the mean

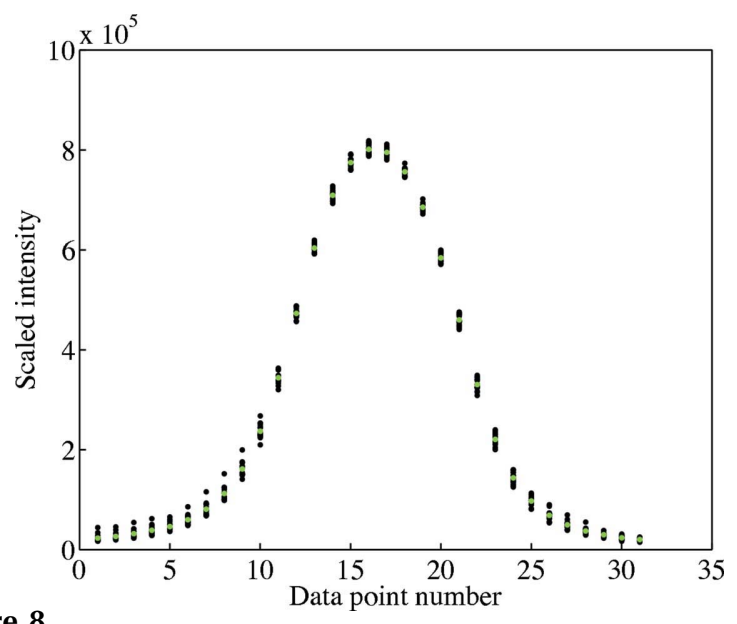

Figure 8

The reference peak shown as green dots is the average of the 15 most intense Bragg peaks all scaled to the intensity of the $(1,1,1)$ reflection.

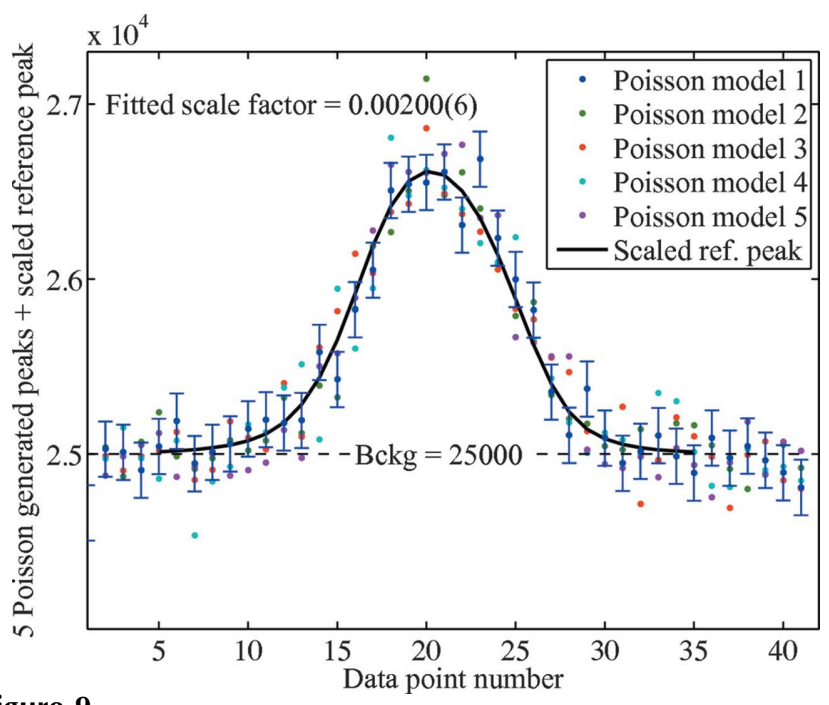

Figure 9

Generated model peaks using Poisson randomization, all having a generated scale factor of 0.002 . The centered $n=31$ data points of the reference peak, scaled by $a=0.002$ and with the background level of 25000 counts added, is shown as the black line. 
as well as the standard deviation of $a$ is readily derived. The mean is of course close to the chosen value of the scale factor, and for this weak peak the relative standard deviation on $a$ is $1.2 \%$. One can repeat this analysis for a whole range of chosen scale factors between 0.001 up to 10 and it turns out that the relative standard deviation on $a$ is approximated well by the algorithm

$$
\log _{10}[s d(a) / a]=\log _{10}[p 1 a+p 2],
$$

with $p 1=-0.76$ (6) and $p 2=-3.5$ (1). This result is used for data points (crosses) in Fig. 6.

\section{APPENDIX $B$ Multiple peak fitting with common line shape}

The three peaks in Fig. 10 represent an X-ray diffraction pattern given by the data set $y_{i}, i=1,2, \ldots, N$. The peaks all have the same shape, centered at position $x_{j}, j=1,2,3$, as given by the function $f$, but their peak heights $p_{j}, j=1,2,3$, should be determined by minimizing $F=\sum_{i}^{N}\left[y_{i}-p_{j} f\left(x_{i}-x_{j}\right)\right]^{2}$. The partial derivatives of $F$ with respect to the parameters $p_{j}$ must therefore vanish, leading to $j$ linear equations with $j$ unknowns, the so-called normal equations, which in matrix form read

$$
\left\{M_{j k}\right\}\left\{p_{j}\right\}=\left\{b_{j}\right\}
$$

with the elements

$$
M_{j k}=\sum_{i=1}^{N} f\left(x_{i}-x_{j}\right) f\left(x_{i}-x_{k}\right)
$$

and

$$
b_{k}=\sum_{i=1}^{N} y_{i} f\left(x_{i}-x_{k}\right) .
$$

Note that in the case of non-overlapping peaks as discussed in the main text of this paper, the matrix $M_{j k}$ has only non-

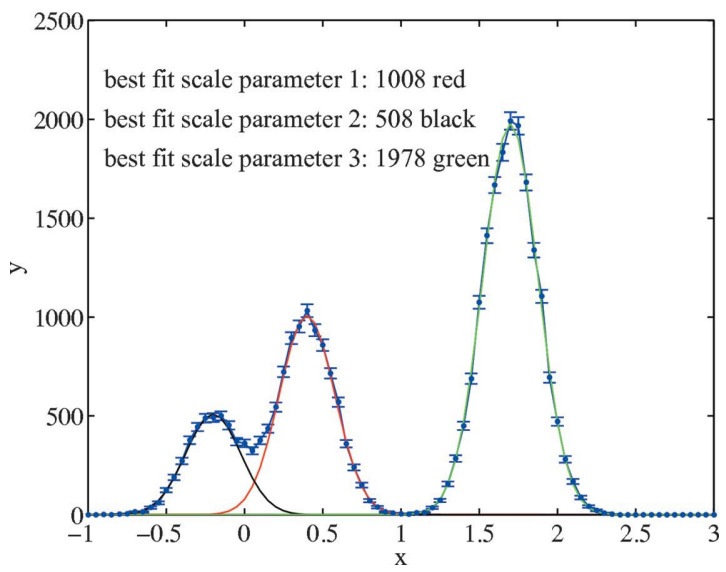

Figure 10

A data set with error bars, generated by randomizing three Gaussian peaks with different amplitudes and different centers, but all with the same peak shape. Whether the generating peaks are Gaussian or are generated by any other means, analytic or numeric, is irrelevant for the illustration, as long as they all have the same shape. vanishing elements in the diagonal and they are all identical. In that case the solution is reduced to

$$
p_{j}=\sum_{i=1}^{N} y_{i} f\left(x_{i}-x_{j}\right) / M_{j j}
$$

which is the same as the single-peak determination given in $\S 2.4$.

Fig. 10 illustrates the solution. The 'data' are synthesized by Poisson-randomizing three Gaussians with amplitudes 500, 1000 and 2000, centered at $x_{1}=-0.2, x_{2}=0.4$ and $x_{3}=1.8$, respectively. The solution for the amplitudes, $p_{1}=508, p_{2}=$ 1008 and $p_{3}=1978$, are indeed in accordance with the generating amplitudes.

The theoretically calculated structure factors depicted in Fig. 1 were kindly provided by Lasse Bjerg, Aahus University. We acknowledge fruitful discussions with Niels Bindzus and Bo Brummerstedt Iversen, Aarhus University. The diffractometer was financed by Carlsberg Foundation. Travel expenses were covered by DanScatt.

\section{References}

Alexander, L., Klug, H. P. \& Kummer, E. (1948). J. Appl. Phys. 19, 742.

Bindzus, N., Straas $\varnothing$, T., Wahlberg, N., Becker, J., Bjerg, L., Dippel, A.-C. \& Iversen, B. B. (2014). Acta Cryst. A70, doi:10.1107/ S2053273313026600.

Blaha, P., Schwarz, K., Madsen, G. K. H., Kvasnicka, D. \& Luitz, J. (2008). WIEN2k, An Augmented Plane Wave + Local Orbitals Program for Calculating Crystal Properties. Technische Universität, Wien, Austria.

Cheary, R. W. \& Coelho, A. (1992). J. Appl. Cryst. 25, 109-121.

Cooper, M. J. \& Glasspool, A. V. (1976). J. Appl. Cryst. 9, 63-67.

Coppens, P. (1997). X-ray Charge Densities and Chemical Bonding. Oxford University Press.

De Wolff, P. M. (1959). Appl. Sci. Res. 7, 102-112.

Dippel, A.-C. et al. (2014). In preparation.

Fischer, A., Tiana, D., Scherer, W., Batke, K., Eickerling, G., Svendsen, H., Bindzus, N. \& Iversen, B. B. (2011). J. Phys. Chem. A, 115, 13061-13071.

Hepp, A. \& Baerlocher, C. (1988). Aust. J. Phys. 41, 229.

Ida, T. \& Izumi, F. (2011). J. Appl. Cryst. 44, 921-927.

International Tables of Crystallography (1992). Vol. C. Dordrecht: Kluwer.

Laar, B. van \& Yelon, W. B. (1984). J. Appl. Cryst. 17, 47-54.

Lucas, B. W. (1969). Acta Cryst. A25, 627-631.

McCusker, L. B., Von Dreele, R. B., Cox, D. E., Louër, D. \& Scardi, P. (1999). J. Appl. Cryst. 32, 36-50.

McSkimin, H. J. \& Andreatch, P. (1972). J. Appl. Phys. 43, 2944-2948.

Nishibori, E., Sunaoshi, E., Yoshida, A., Aoyagi, S., Kato, K., Takata, M. \& Sakata, M. (2007). Acta Cryst. A63, 43-52.

Pawley, G. S. (1981). J. Appl. Cryst. 14, 357-361.

Spackman, M. A. (1991). Acta Cryst. A47, 420-427.

Stewart, R. F. (1973). Acta Cryst. A29, 602-605.

Straas $\varnothing$, T., Becker, J., Iversen, B. B. \& Als-Nielsen, J. (2013). J. Synchrotron Rad. 20, 98-104.

Svendsen, H., Overgaard, J., Busselez, R., Arnaud, B., Rabiller, P., Kurita, A., Nishibori, E., Sakata, M., Takata, M. \& Iversen, B. B. (2010). Acta Cryst. A66, 458-469.

Taylor, J. R. (1997). An Introduction to Error Analysis, 2nd ed. Sausalito: University Science Books.

Yamamoto, K., Takahashi, Y., Ohshima, K., Okamura, F. P. \& Yukino, K. (1996). Acta Cryst. A52, 606-613. 